Ciencia y tecnología 


\section{Materiales bioinorgánicos de paladio y cobre con compuestos orgánicos de actividad antitumoral}

Wilfredo Hernández Gorritti, Juan Paz Castillo, Abraham Vaisberg, Evgenia Spodine, Rainer Richter, Lothar Beyer ${ }^{1}$

Ingeniería Industrial n. 27, 2009, ISSN 1025-9929, pp. 237-257.

RESUMEN: En el presente trabajo se informa acerca de la preparación y caracterización de los compuestos orgánicos derivados del furaldehído tiosemicarbazona y sus respectivos complejos metálicos de paladio(II) y cobre(II). El análisis elemental y las técnicas espectroscópicas confirman las fórmulas estructurales propuestas para los ligantes orgánicos y sus respectivos complejos de paladio(II) y cobre(II). La actividad biológica in vitro de los ligantes y los complejos metálicos reveló que los complejos de paladio(II), $\mathrm{Pd}\left(\mathrm{LF}^{4}\right)_{2}$ y $\mathrm{Pd}\left(\mathrm{LF}^{5}\right)_{2}$ presentan mayor actividad citotóxica in vitro $\left(\mathrm{CI}_{50}=0.28-1.23 \mu \mathrm{M}\right)$ con respecto a los ligantes frente a diferentes líneas de células tumorales de humano.

Palabras clave: Tiosemicarbazona / complejos de paladio(II) y cobre(II) / actividad citotóxica antitumoral / crecimiento celular

\section{Bioinorganic materials of palladium(III) and copper(II) with organic compounds of antitumor activity}

ABSTRACT: The present work informs about the preparation and characterization of organic compounds derived from furaldehyde thiosemicarbazone and their respective palladium(II) and copper(II) complexes. The elemental analysis and spectroscopic techniques confirm the proposed structural formula for the organic ligands and their respective palladium(II) and copper(II) complexes. The in vitro biological activity of ligands and metal complexes revealed that the palladium (II) complexes, $\mathrm{Pd}\left(\mathrm{LF}^{4}\right)_{2}$ and $\mathrm{Pd}$ $\left(\mathrm{LF}^{5}\right)_{2}$, have a higher in vitro cytototoxic activity $\left(\mathrm{CI}_{50}=0.28-1.23 \mu \mathrm{M}\right)$ with respect to ligands against different cell lines of human tumor.

Keywords: Furaldehyde thiosemicarbazone / Palladium(II) and copper(II) complexes / antitumor cytotoxic activity / cell growth

1 Los autores expresan su agradecimiento al laboratorio de investigación y desarrollo 


\section{INTRODUCCIÓN}

Como parte del trabajo de investigación relacionado con la actividad antitumoral de los complejos metálicos con ligantes orgánicos derivados de la tiosemicarbazona, se ha evaluado la actividad citotóxica in vitro de los complejos de paladio(II) con compuestos orgánicos del pirrol tiosemicarbazona y tiofeno tiosemicarbazona. Los complejos de paladio(II) preparados (figura 1) presentaron mayores actividades citotóxicas frente a diferentes líneas de células tumorales al obtener bajas concentraciones de inhibición $\left(\mathrm{CI}_{50}=4.5-55.5 \mu \mathrm{M}\right)$ con respecto a sus ligantes orgánicos $\left(\mathrm{CI}_{50}=324.0-54.0 \mu \mathrm{M}\right)$. Estos resultados indican que la citotoxicidad se incrementa cuando los ligantes orgánicos se encuentran coordinados al metal paladio. ${ }^{2}$

Dentro de los complejos de paladio(II) ensayados biológicamente, el complejo $\mathrm{Pd}\left(\mathrm{TSC}^{4}\right)_{2}$ resultó ser el más citotóxico frente a las líneas ce-

\section{Figura 1}

Fórmulas estructurales de los complejos de paladio(II) con ligantes pirrol tiosemicarbazona y tiofeno tiosemicarbazona
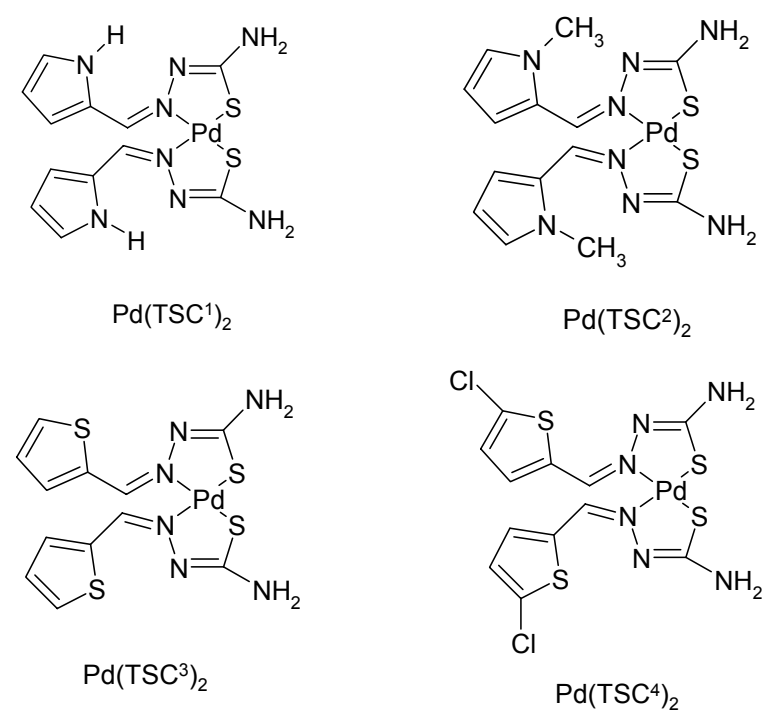

de la Facultad de Ciencias y Filosofía de la Universidad Peruana Cayetano Heredia por la evaluación de la actividad citotóxica de los compuestos preparados.

2 Hernández, W. Bioinorg. Chem. Appl., 2008. 
lulares tumorales DU145, MCF-7, HT-29 y PC3 con valores de $\mathrm{CI}_{50}$ de $15.16,9.10,13.65$ y $4.55 \mu \mathrm{M}$, respectivamente.

La actividad citotóxica de los compuestos derivados de la hidrazona con grupos sustituyentes 5-nitro-2-furil-2 tiazol (figura 2) reveló un mayor grado de citotoxicidad sobre el crecimiento tumoral de células del adenocarcinoma mamario de ratón. ${ }^{3}$

\section{Figura 2}

Fórmulas estructurales de los ligantes derivados del 5-nitro-furaldehído<smiles>CC(C)=NNc1nc(-c2ccc([N+](=O)[O-])o2)cs1</smiles><smiles>O=CNNc1nc(-c2ccc([N+](=O)[O-])o2)cs1</smiles>

Se ha informado que derivados de la fenilhidrazona con grupos 5nitro-2-furaldehído (figura 3) presentan una actividad antitumoral in vitro a bajas concentraciones micromolares de inhibición frente a las células leucémicas L-1210 de murino. ${ }^{4}$

\section{Figura 3}

Fórmula estructural de derivados de fenilhidrazona con ligantes 5-nitro-2-furaldehído<smiles>[X]c1ccc(N/N=C/c2ccc([N+](=O)[O-])o2)cc1</smiles>

Otros derivados de la fenilhidrazona con grupos 5-nitro-2-furaldehído y guanidina (figura 4) presentan una actividad citotóxica in vitro a bajas concentraciones micromolares de inhibición $\left(\mathrm{CI}_{50}=0.3-3.0 \mu \mathrm{M}\right)$ frente diferentes líneas de células tumorales de leucemia, próstata, colon, riñón, ovario y melanoma de humano. ${ }^{5}$

Morris, J. et al. Center Reserch, 29, 1969, p. 2145.

Newman, R. et al. Journal of Pharmaceutical Scientes 71, 1981, p. 831.

Brozowski, Z. et. al. European journal of medicinal chemistry 42, 2007, p. 1218. 


\section{Figura 4}

Fórmula estructural de la fenilhidrazona con ligantes 5-nitro-furaldehído y guanidina<smiles>C=CCN/C(=N\C(=O)c1cc(C(=O)Nc2ccc(Cl)cc2)c(Cl)cc1C)N/N=C/c1ccc([N+](=O)[O-])o1</smiles>

Se ha evaluado la actividad citotóxica de los complejos de cobre(II) y cobalto(II) con ligantes furfuraldehído semicarbazona y furfuraldehído tiosemicarbazona (figura 5) en células tumorales de pulmón MB9812 de humano. Los resultados indicaron que la citotoxicidad de los complejos metálicos $\left(\mathrm{CI}_{50}=25.0-100.0 \mu \mathrm{M}\right)$ fue mayor con respecto a sus ligantes orgánicos. Además, estos complejos metálicos por la geometría molecular cuadrada plana que presentan pueden intercalarse con las bases nitrogenadas del ADN celular tumoral, logrando de esta manera inhibir la síntesis del ADN. ${ }^{6}$

Figura 5

Complejos de cobre(II) y cobalto(II) con ligantes derivados de furaldehído semicarbazonas (a) y furaldehído tiosemicarbazona (b)<smiles></smiles>

(a)<smiles>[X]c1ccoc1C=NN1N=C(N)S[N+]12SC(N)=NN2C=Cc1ccco1</smiles>

$\mathrm{M}=\mathrm{Cu}, \mathrm{Co}$

(b)

Con los antecedentes antes mencionados, se llevó a cabo la preparación y caracterización de los ligantes orgánicos derivados del fural-

6 Hall, I. et al. Pharmazie 55, 2000, p. 937. 
dehído tiosemicarbazona de fórmula global, $\mathrm{X}-\mathrm{C}_{4} \mathrm{H}_{2} \mathrm{O}-\mathrm{CH}=\mathrm{N}-\mathrm{NHC}(\mathrm{S})$ $\mathrm{NHR} \quad\left[\mathrm{X}=\mathrm{H}, \mathrm{Ph}, \mathrm{Et},-\mathrm{NO}_{2},-\mathrm{Cl} ; \mathrm{R}=\mathrm{H}, \mathrm{Ph}\right]$ y sus respectivos complejos metálicos de paladio(II) y cobre(II). La actividad biológica de los compuestos preparados se realizó in vitro frente a diferentes líneas de células tumorales de humano.

\section{OBJETIVOS}

\subsection{Objetivo general}

El objetivo general del trabajo ha sido la preparación y caracterización de complejos de paladio(II) y cobre(II) con ligantes orgánicos derivados del furaldehído tiosemicarbazona y la evaluación de su actividad biológica en células tumorales de humano.

\subsection{Objetivos específicos}

Los objetivos específicos se pueden dividir en:

- Preparación y caracterización de los ligantes orgánicos mediante análisis elemental y técnicas espectroscópicas de IR, RMN-( ${ }^{1} \mathrm{H}$ y $\left.{ }^{13} \mathrm{C}\right)$.

- Preparación y caracterización de los complejos de paladio(II) mediante técnicas espectroscópicas de IR y RMN $-{ }^{1} \mathrm{H}$.

- Preparación y caracterización de los complejos de cobre(II) mediante técnicas espectroscópicas de IR.

- Evaluación de la actividad citotóxica de los ligantes orgánicos y los complejos de paladio(II) y cobre(II) frente a diferentes líneas de células tumorales de humano.

\section{MÉTODO EXPERIMENTAL}

En la preparación de los ligantes orgánicos y sus respectivos complejos de paladio(II) y cobre(II) se ha empleado el método químico que involucra reacciones químicas, separación, purificación y recristalización de los productos preparados en diferentes solventes orgánicos. 


\subsection{Síntesis de ligantes orgánicos, $\mathrm{LF}^{1-7}$}

Los ligantes derivados del furaldehído tiosemicarbazona (TSC $\left.{ }^{1-7}\right)$ fueron preparados de acuerdo con los métodos informados, tal como se muestra en el esquema $1 .^{7}$

\section{Esquema 1}

Preparación de los ligantes orgánicos derivados de furaldehído tiosemicarbazona<smiles>[X]c1ccc(C=O)o1</smiles>

Furaldehído<smiles>[R]NC(=S)N([2H])N</smiles>

Tiosemicarbazida
Etanol reflujo, $4 \mathrm{~h}$ $-\mathrm{H}_{2} \mathrm{O}$<smiles></smiles>

Ligante tiosemicarbazona

$$
\begin{aligned}
& L^{1}(X=H, R=H) \\
& L F^{2}(X=\mathrm{Cl}, \mathrm{R}=\mathrm{H}) \\
& \mathrm{LF}^{3}(\mathrm{X}=\mathrm{Et}, \mathrm{R}=\mathrm{H}) \\
& \mathrm{LF}^{4}(\mathrm{X}=\mathrm{H}, \mathrm{R}=\mathrm{Ph}) \\
& \mathrm{LF}^{5}(\mathrm{X}=\mathrm{Ph}, \mathrm{R}=\mathrm{Ph}) \\
& \mathrm{LF}^{6}(\mathrm{X}=\mathrm{Et}, \mathrm{R}=\mathrm{Ph}) \\
& \operatorname{LF}^{7}(\mathrm{X}=\mathrm{Cl}, \mathrm{R}=\mathrm{Ph})
\end{aligned}
$$

Una solución del furaldehído $(20 \mathrm{mmol})$ en etanol $(50 \mathrm{~mL})$ se adiciona gota a gota a $100 \mathrm{~mL}$ de una solución etanólica de tiosemicarbazida (20 mmol) bajo agitación constante. Luego, la mezcla se lleva a reflujo por 4 horas y se mantiene agitada por 2 horas adicionales a temperatura ambiente. El producto sólido obtenido se separa por filtración y se lava varias veces con agua y etanol frío. Finalmente, el producto se seca al vacío durante ocho horas. Los ligantes $\mathrm{LF}^{6}$ y $\mathrm{LF}^{7}$ no precipitaron como sólidos y se obtuvieron en solución al final de la reacción. Las soluciones de estos ligantes fueron concentradas en el evaporador rotatorio hasta la mitad de sus volúmenes y, finalmente, se dejaron evaporar lentamente a temperatura ambiente.

En la figura 6 se muestra a los ligantes orgánicos obtenidos, $\mathrm{LF}^{5}$ y $\mathrm{LF}^{6}$, en forma de agujas y cristales rectangulares, respectivamente.

7 Lobana, T. et al. J. Chem. Doc, Dalton Trans, 1997, p. 4289. 
Figura 6

Ligantes de los derivados del furaldehído tiosemicarbazona preparados

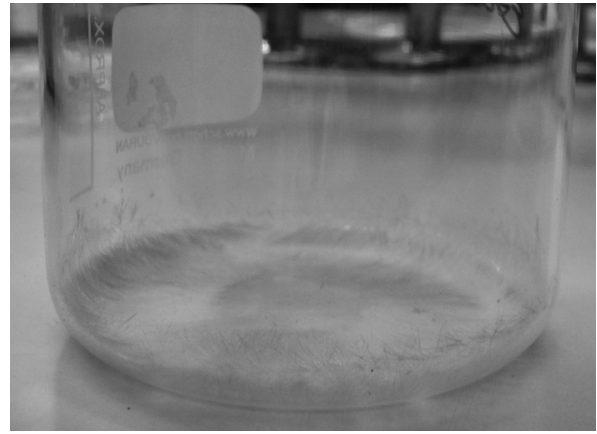

4-N-fenil-5'-fenilfuraldehído tiosemicarbazona $\left(\mathrm{LF}^{5}\right)$

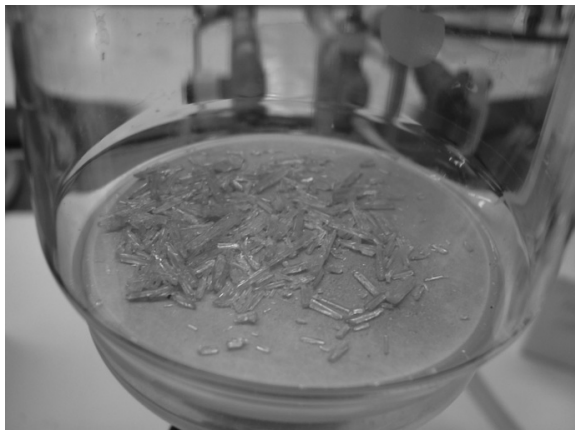

4-N-etil-5'-fenilfuraldehído tiosemicarbazona $\left(\mathrm{LF}^{6}\right)$

Los rendimientos y caracterización de los compuestos preparados están resumidos en las tablas 1-4. Los ligantes fueron caracterizados por análisis elemental (tabla 1), espectroscopía de IR (tabla 2) y RMN $\left({ }^{1} \mathrm{H} \mathrm{y}{ }^{13} \mathrm{C}\right)$ (tablas 3 y 4$)$.

Tabla 1

Rendimientos y resultados del análisis elemental [\% exp.(calc.)] de los ligantes orgánicos sintetizados

\begin{tabular}{lrrrrrr}
\hline \multicolumn{2}{c}{$\begin{array}{c}\text { Compuesto Rendimiento } \\
(\%)\end{array}$} & $\begin{array}{c}\text { Fórmula } \\
\text { general/(PM) }\end{array}$ & \%C & \%H & \%N & $\% \mathbf{S}$ \\
\hline $\mathrm{LF}^{1}$ & 85,74 & $\mathrm{C}_{6} \mathrm{H}_{7} \mathrm{~N}_{3} \mathrm{OS}$ & 42,70 & 4,30 & 24,62 & 18,95 \\
& & $(169,21)$ & $(42,59)$ & $(4,17)$ & $(24,83)$ & $(18,45)$ \\
$\mathrm{LF}^{2}$ & 79,86 & $\mathrm{C}_{6} \mathrm{H}_{6} \mathrm{~N}_{3} \mathrm{OSCl}$ & 35,69 & 2,67 & 20,45 & 15,46 \\
& & $(203,65)$ & $(35,39)$ & $(2,97)$ & $(20,63)$ & $(15,74)$ \\
$\mathrm{LF}^{3}$ & 74,58 & $\mathrm{C}_{8} \mathrm{H}_{11} \mathrm{~N}_{3} \mathrm{OS}$ & 48,98 & 5,87 & 21,35 & 16,66 \\
& & $(197,26)$ & $(48,71)$ & $(5,62)$ & $(21,30)$ & $(16,25)$ \\
$\mathrm{LF}^{4}$ & 81,70 & $\mathrm{C}_{12} \mathrm{H}_{11} \mathrm{~N}_{3} \mathrm{OS}$ & 58,81 & 4,64 & 17,26 & 12,91 \\
& & $(245,30)$ & $(58,76)$ & $(4,52)$ & $(17,13)$ & $(13,07)$ \\
$\mathrm{LF}^{5}$ & 62,20 & $\mathrm{C}_{18} \mathrm{H}_{15} \mathrm{~N}_{3} \mathrm{OS}$ & 67,00 & 4,94 & 13,33 & 9,81 \\
& & $(321,40)$ & $(67,27)$ & $(4,70)$ & $(13,07)$ & $(9,98)$ \\
$\mathrm{LF}^{6}$ & 68,81 & $\mathrm{C}_{14} \mathrm{H}_{15} \mathrm{~N}_{3} \mathrm{OS}$ & 61,34 & 5,59 & 15,54 & 10,89 \\
& & $(273,36)$ & $(61,51)$ & $(5,53)$ & $(15,37)$ & $(11,73)$ \\
$\mathrm{LF}^{7}$ & 58,65 & $\mathrm{C}_{12} \mathrm{H}_{10} \mathrm{~N}_{3} \mathrm{OSCl}$ & 51,11 & 3,72 & 15,04 & 11,00 \\
& & $(279,75)$ & $(51,52)$ & $(3,60)$ & $(15,02)$ & $(11,46)$ \\
\hline
\end{tabular}


Tabla 2

Frecuencias vibracionales $\left(\mathrm{v}\left(\mathrm{cm}^{-1}\right)\right)$ en el infrarrojo de los ligantes orgánicos sintetizados*

\begin{tabular}{lrrrr}
\hline Compuesto & $v\left(-\mathrm{NH}_{2}\right)$ & $v(-\mathrm{NH})$ & $v(\mathrm{C}=\mathrm{N})$ & $v(\mathrm{C}=\mathbf{S})$ \\
\hline $\mathrm{LF}^{1}$ & $3.413(\mathrm{~m})$ & $3.143(\mathrm{i})$ & $1.610(\mathrm{i})$ & $843(\mathrm{~m})$ \\
& $3.224(\mathrm{~d})$ & & & \\
$\mathrm{LF}^{2}$ & $3.417(\mathrm{~m})$ & $3.195(\mathrm{~d})$ & $1.595(\mathrm{i})$ & $847(\mathrm{~m})$ \\
& $3.271(\mathrm{~d})$ & & & \\
$\mathrm{LF}^{3}$ & $3.356(\mathrm{~d})$ & $3.161(\mathrm{~d})$ & $1.597(\mathrm{~m})$ & $859(\mathrm{~d})$ \\
& $3.327(\mathrm{~d})$ & & & \\
$\mathrm{LF}^{4}$ & $3.296(\mathrm{~m}, \mathrm{NPh})$ & $3.132(\mathrm{~m})$ & $1.620(\mathrm{~d})$ & $924(\mathrm{~d})$ \\
$\mathrm{LF}^{5}$ & $3.290(\mathrm{~d}, \mathrm{NPh})$ & $3.159(\mathrm{~d})$ & $1.598(\mathrm{~m})$ & $1.028(\mathrm{~d})$ \\
$\mathrm{LF}^{6}$ & $3.319(\mathrm{~m}, \mathrm{NPh})$ & $3.147(\mathrm{~m})$ & $1.595(\mathrm{~d})$ & $970(\mathrm{~d})$ \\
$\mathrm{LF7}$ & $3.325(\mathrm{~m}, \mathrm{NPh})$ & $3.163(\mathrm{~m})$ & $1.599(\mathrm{~d})$ & $1.018(\mathrm{~d})$ \\
\hline
\end{tabular}

${ }^{*} \mathrm{i}$ = banda intensa, $\mathrm{m}$ = banda media, $\mathrm{d}=$ banda débil.

Tabla 3

Datos de espectroscopía de RMN-1 $\mathrm{H}$ (ppm) de los ligantes orgánicos derivados del furaldehído tiosemicarbazona

\begin{tabular}{|c|c|c|c|c|}
\hline Compuesto & $\delta(-\mathrm{CH}=\mathrm{N})$ & $\delta(-\mathrm{NH})$ & $\delta($ NHR), & $\delta$ (Furano), $\delta$ (Fenilo) \\
\hline \multirow[t]{3}{*}{$\mathrm{LF}^{1}$} & $7,78(\mathrm{~s}, 1 \mathrm{H})$ & $10,60(\mathrm{~s}, 1 \mathrm{H})$ & $6,40,7,31$ & $6,76\left(d, H^{3}\right)$ \\
\hline & & & $\left(\mathrm{d}, 2 \mathrm{H}, \mathrm{NH}_{2}\right)$ & $6,53\left(t, H^{4}\right)$ \\
\hline & & & & $7,60\left(\mathrm{~d}, \mathrm{H}^{5}\right)$ \\
\hline \multirow[t]{2}{*}{$\mathrm{LF}^{2}$} & $8,10(\mathrm{~s}, 1 \mathrm{H})$ & $11,47(\mathrm{~s}, 1 \mathrm{H})$ & $8,21,7,61$ & $7,10\left(\mathrm{~d}, \mathrm{H}^{3}\right)$ \\
\hline & & & $\left(\mathrm{d}, 2 \mathrm{H}, \mathrm{NH}_{2}\right)$ & $7,29\left(\mathrm{~d}, \mathrm{H}^{4}\right)$ \\
\hline \multirow[t]{4}{*}{$\mathrm{LF}^{3}$} & $7,88(\mathrm{~s}, 1 \mathrm{H})$ & $11,35(\mathrm{~s}, 1 \mathrm{H})$ & $7,54,8,15$ & $6,84\left(\mathrm{~d}, \mathrm{H}^{3}\right)$ \\
\hline & & & $\left(\mathrm{d}, 2 \mathrm{H}, \mathrm{NH}_{2}\right)$ & $6,24\left(\mathrm{~d}, \mathrm{H}^{4}\right)$ \\
\hline & & & & $2,65\left(\mathrm{c},-\mathrm{CH}_{2}\right)$ \\
\hline & & & & $1,17\left(\mathrm{t},-\mathrm{CH}_{3}\right)$ \\
\hline \multirow[t]{6}{*}{$\mathrm{LF}^{4}$} & $8,08(\mathrm{~d}, 1 \mathrm{H})$ & $11,97(\mathrm{~s}, 1 \mathrm{H})$ & 9,86 & $6,65\left(t, H^{4}\right)$ \\
\hline & & & (s, 1H, NHPh) & $7,08\left(\mathrm{~d}, \mathrm{H}^{3}\right)$ \\
\hline & & & & $7,85\left(\mathrm{~d}, \mathrm{H}^{5}\right)$ \\
\hline & & & & $7,18\left(\mathrm{t}, 1 \mathrm{H}_{\text {meta }}, \mathrm{Ph}\right)$ \\
\hline & & & & $7,35\left(\mathrm{t}, 2 \mathrm{H}_{\text {para }}, \mathrm{Ph}\right)$ \\
\hline & & & & $7,57\left(\mathrm{~d}, 2 \mathrm{H}_{\text {orto }}, \mathrm{Ph}\right)$ \\
\hline
\end{tabular}


(continuación)

\begin{tabular}{|c|c|c|c|c|}
\hline Compuesto & $\delta(-\mathrm{CH}=\mathrm{N})$ & $\delta(-\mathrm{NH})$ & $\delta(\mathrm{NHR})$, & $\delta$ (Furano), $\delta$ (Fenilo) \\
\hline \multirow[t]{5}{*}{$\mathrm{LF}^{5}$} & $8,10(\mathrm{~s}, 1 \mathrm{H})$ & $11,92(\mathrm{~s}, 1 \mathrm{H})$ & 9,95 & $7,16\left(\mathrm{dd}, \mathrm{H}^{4}\right)$ \\
\hline & & & $(\mathrm{s}, 1 \mathrm{H}, \mathrm{NHPh})$ & $7,21\left(\mathrm{~d}, \mathrm{H}^{3}\right)$ \\
\hline & & & & 7,242(m,5H,C-Ph) \\
\hline & & & & $7,60\left(\mathrm{~d}, 2 \mathrm{H}_{\text {para, }} \mathrm{N}-\mathrm{Ph}\right)$ \\
\hline & & & & $7,83\left(\mathrm{~d}, 2 \mathrm{H}_{\text {orto, }}, \mathrm{N}-\mathrm{Ph}\right)$ \\
\hline \multirow[t]{7}{*}{$\mathrm{LF}^{6}$} & $8,01(\mathrm{~s}, 1 \mathrm{H})$ & $11,77(\mathrm{~s}, 1 \mathrm{H})$ & 9,78 & $6,29\left(\mathrm{~d}, \mathrm{H}^{4}\right)$ \\
\hline & & & $(\mathrm{s}, 1 \mathrm{H}, \mathrm{NHPh})$ & $6,97\left(\mathrm{~d}, \mathrm{H}^{3}\right)$ \\
\hline & & & & $1,19\left(\mathrm{t},-\mathrm{CH}_{3}\right)$ \\
\hline & & & & $2,68\left(\mathrm{c},-\mathrm{CH}_{2}\right)$ \\
\hline & & & & $7,18\left(\mathrm{t}, 2 \mathrm{H}_{\text {meta }}, \mathrm{N}-\mathrm{Ph}\right)$ \\
\hline & & & & $7,36\left(\mathrm{t}, 1 \mathrm{H}_{\text {para, }} \mathrm{N}-\mathrm{Ph}\right)$ \\
\hline & & & & $7,59\left(\mathrm{~d}, 2 \mathrm{H}_{\text {orto, }}, \mathrm{N}-\mathrm{Ph}\right)$ \\
\hline
\end{tabular}

Tabla 4

Datos de espectroscopía de $\mathrm{RMN}^{-13} \mathrm{C}(\mathrm{ppm})$ de los ligantes derivados del furaldehído tiosemicarbazona

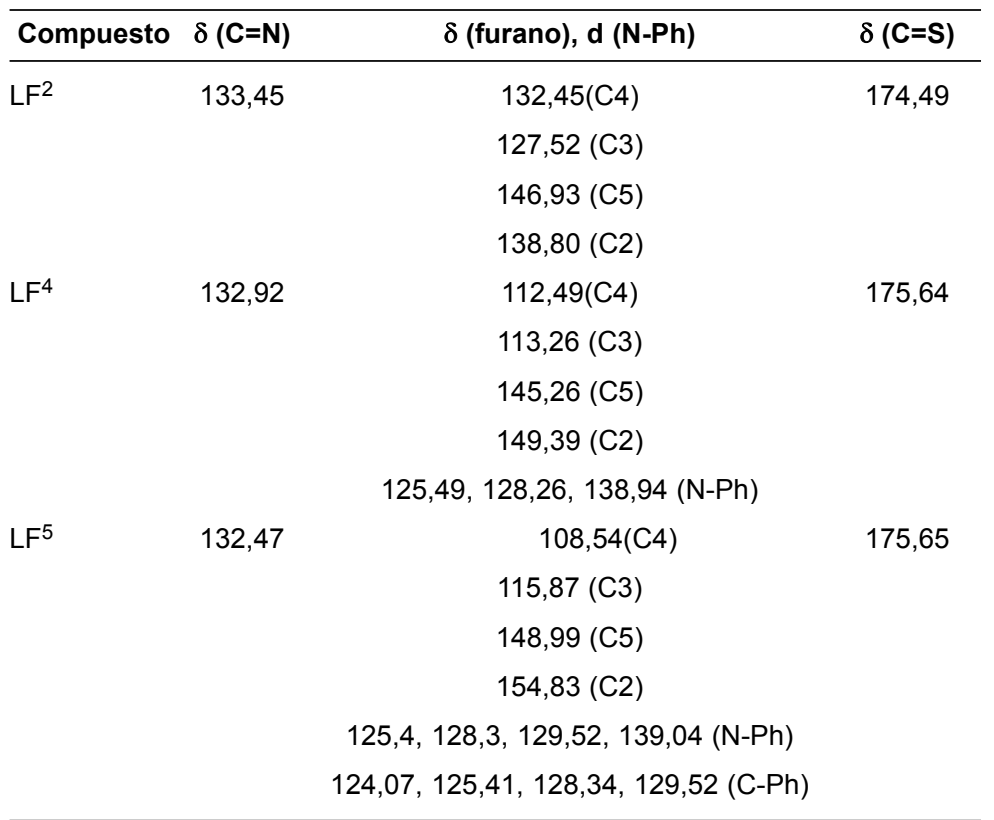

(continúa) 


\begin{tabular}{lccc} 
(continuación) & & & \\
\hline Compuesto & $\delta(\mathbf{C}=\mathbf{N})$ & $\delta$ ( furano), $\mathbf{d}$ ( N-Ph) & $\delta$ (C=S) \\
\hline $\mathrm{LF}^{6}$ & 133,13 & $11,88\left(\mathrm{CH}_{3}\right)$ & 175,43 \\
& & $21,05\left(\mathrm{CH}_{2}\right)$ & \\
& $114,81(\mathrm{C} 3)$ & \\
& $107,42(\mathrm{C} 4)$ & \\
& $147,71(\mathrm{C} 5)$ & \\
& & $160,11(\mathrm{C} 2)$ &
\end{tabular}

3.2 Síntesis de los complejos de paladio(II) con ligantes derivados del furaldehído tiosemicarbazona, $\mathrm{Pd}\left(\mathrm{LF}^{1-7}\right)_{2}$ (Esquema 2)

Una solución de la sal acetoacetonato de paladio(II) $(2.0 \mathrm{mmol})$ en diclorometano-etanol $(1: 1,50 \mathrm{~mL})$ se adiciona gota a gota durante 30 minutos a una solución caliente de furaldehído tiosemicarbazona $(4.0 \mathrm{mmol})$ en acetona-etanol $(2: 1,60 \mathrm{~mL})$ bajo agitación constante. Luego, a la mezcla final se le agrega una solución de acetato de sodio $(2 \mathrm{mmol})$ en agua $(5 \mathrm{~mL})$ y se somete a reflujo por tres horas. Al finalizar la reacción, se forman precipitados de color rojo y anaranjado. La mezcla de reacción se filtra y el sólido obtenido se lava con agua y etanol frío $(50 \mathrm{~mL})$, y luego se seca al vacío durante 12 horas. Luego de la recristalización en $\mathrm{CHCl}_{3}-\mathrm{C}_{2} \mathrm{H}_{5} \mathrm{OH}$, se obtuvieron cristales de buena calidad para los complejos de paladio(II), $\mathrm{Pd}\left(\mathrm{LF}^{4}\right)_{2}$ y $\mathrm{Pd}\left(\mathrm{LF}^{5}\right)_{2}$, Rendimiento: $\operatorname{Pd}\left(\mathrm{LF}^{1}\right)_{2}: 57.24 \%, \operatorname{Pd}\left(\mathrm{LF}^{2}\right)_{2}: 62.35 \%, \mathrm{Pd}_{2}\left(\mathrm{LF}^{3}\right)_{2}: 74.28 \%$, $\mathrm{Pd}\left(\mathrm{LF}^{4}\right)_{2}: 55.69 \%, \mathrm{Pd}\left(\mathrm{LF}^{5}\right)_{2}: 65.75 \%, \mathrm{Pd}\left(\mathrm{LF}^{6}\right)_{2}: 76.94 \%, \mathrm{Pd}\left(\mathrm{LF}^{7}\right)_{2}:$ $52.48 \%$. 
<smiles>[R]NC(=S)N([2H])/N=C/c1ccc([X])o1</smiles>

Furaldehído tiosemicarbazona<smiles></smiles><smiles></smiles>

Acetona-Etanol $3 \mathrm{~h}$, reflujo<smiles>[R]NC1=NN(Cc2ccco2)[P+]2(S1)SC(N[R])=NN2/N=C\c1ccc([X])o1</smiles>

Complejo metálico $\mathrm{Pd}\left(\mathrm{LF}^{1}\right)_{2}(\mathrm{X}=\mathrm{H}, \mathrm{R}=\mathrm{H})$ $\mathrm{Pd}\left(\mathrm{LF}^{2}\right)_{2}(\mathrm{X}=\mathrm{Cl}, \mathrm{R}=\mathrm{H})$ $\mathrm{Pd}\left(\mathrm{LF}^{3}\right)_{2}(\mathrm{X}=\mathrm{Et}, \mathrm{R}=\mathrm{H})$ $\mathrm{Pd}\left(\mathrm{LF}^{4}\right)_{2}(\mathrm{X}=\mathrm{H}, \mathrm{R}=\mathrm{Ph})$ $\mathrm{Pd}\left(\mathrm{LF}^{5}\right)_{2}(\mathrm{X}=\mathrm{Ph}, \mathrm{R}=\mathrm{Ph})$ $\mathrm{Pd}\left(\mathrm{LF}^{6}\right)_{2}(\mathrm{X}=\mathrm{Et}, \mathrm{R}=\mathrm{Ph})$ $\mathrm{Pd}\left(\mathrm{LF}^{7}\right)_{2}(\mathrm{X}=\mathrm{Cl}, \mathrm{R}=\mathrm{Ph})$

3.3 Síntesis de los complejos de cobre(II) con ligantes furaldehído tiosemicarbazona, $\mathrm{Cu}\left(\mathrm{LF}^{3-6}\right)_{2}$ (Esquema 3)

Una solución de acetato de cobre(II), $\mathrm{Cu}\left(\mathrm{CH}_{3} \mathrm{COO}\right)_{2}(1.0 \mathrm{mmol})$, en etanol caliente $(40 \mathrm{~mL})$ se adiciona gota a gota durante 30 minutos a una solución caliente de furaldehído tiosemicarbazona $(2 \mathrm{mmol})$ en acetona-etanol $(60 \mathrm{~mL})$ bajo agitación constante. Luego, a la mezcla final se le agrega una solución de acetato de sodio ( $2 \mathrm{mmol})$ en agua $(5 \mathrm{~mL})$ y se somete a reflujo por 2 horas. Al finalizar la reacción, se forma un precipitado de color marrón. La mezcla de reacción se filtra y el sólido se lava con agua y etanol frío $(50 \mathrm{~mL})$, y luego se seca al vacío durante 12 horas. Después de la recristalización en $\mathrm{CH}_{2} \mathrm{Cl}_{2}$ $\mathrm{CH}_{3} \mathrm{OH}$, se obtuvieron cristales en forma de agujas cortas muy finas para los complejos $\mathrm{Cu}\left(\mathrm{LF}^{4}\right)_{2}$ y $\mathrm{Cu}\left(\mathrm{LF}^{6}\right)_{2}$. Rendimiento: $\mathrm{Cu}\left(\mathrm{LF}^{3}\right)_{2}$ : $61.58 \%, \mathrm{Cu}\left(\mathrm{LF}^{4}\right)_{2}: 80.91 \%, \mathrm{Cu}\left(\mathrm{LF}^{5}\right)_{2}: 56.35 \%$ y $\mathrm{Cu}\left(\mathrm{LF}^{6}\right)_{2}: 64.28 \%$. 
<smiles>[R]NC1=NN(/C=C\c2ccco2)[C@]2(S1)SC(N[R])=NN2/N=C\c1ccc([X])o1</smiles>

Complejo metálico

$\mathrm{Cu}\left(\mathrm{LF}^{3}\right)_{2}(\mathrm{X}=\mathrm{Et}, \mathrm{R}=\mathrm{H})$

$\mathrm{Cu}\left(\mathrm{LF}^{4}\right)_{2}(\mathrm{X}=\mathrm{H}, \mathrm{R}=\mathrm{Ph})$

$\left.\mathrm{CuLF}^{5}\right)_{2}(\mathrm{X}=\mathrm{Ph}, \mathrm{R}=\mathrm{Ph})$

$\mathrm{Cu}\left(\mathrm{LF}^{6}\right)_{2}(\mathrm{X}=\mathrm{Et}, \mathrm{R}=\mathrm{Ph})$

Las frecuencias vibracionales $\left(\mathrm{v}\left(\mathrm{cm}^{-1}\right)\right)$ en el infrarrojo (IR) y los desplazamientos químicos $(\delta$, ppm) (RMN) se muestran en las tablas 5 y 6 , respectivamente, para los complejos de paladio(II) y cobre(II) obtenidos.

Tabla 5

Frecuencias vibracionales $\left(v\left(\mathrm{~cm}^{-1}\right)\right)$ en el infrarrojo de los complejos de $\mathrm{Pd}$ (II) y Cu(II) sintetizados*

\begin{tabular}{|c|c|c|c|}
\hline Compuesto & $v\left(-\mathrm{NH}_{2}\right)$ & $v(\mathbf{C}=\mathbf{N})$ & $v(\mathbf{C}=\mathbf{S})$ \\
\hline \multirow[t]{2}{*}{$\mathrm{CuLF}^{3}$} & $3.456(d)$ & $1.603(d)$ & $831(d)$ \\
\hline & $3.352(d)$ & & \\
\hline CuLF$^{4}$ & 3.427(d,NPh) & $1.608(d)$ & $870(d)$ \\
\hline $\mathrm{PdLF}^{4}$ & $3.415(\mathrm{~d}, \mathrm{NPh})$ & $1.604(d)$ & $877(d)$ \\
\hline CuLF$^{5}$ & $3.317(\mathrm{~d}, \mathrm{NPh})$ & $1.603(d)$ & $877(d)$ \\
\hline PdLF$^{5}$ & 3.421(d,NPh) & $1.601(d)$ & 924(d) \\
\hline CuLF$^{6}$ & 3.338(da,NPh) & $1.604(d)$ & $876(d)$ \\
\hline PdLF7 & $3.386(\mathrm{da}, \mathrm{NPh})$ & $1.603(d)$ & $885(d)$ \\
\hline
\end{tabular}

${ }^{*} \mathrm{i}$ = banda intensa, $\mathrm{m}$ = banda media, $\mathrm{d}=$ banda débil. 
Tabla 6

Datos de espectroscopía de ${ }^{1} \mathrm{H}-\mathrm{RMN}$ (ppm) del complejos de Pd(II) con ligantes derivados del furaldehído tiosemicarbazona sintetizados

\begin{tabular}{|c|c|c|c|c|}
\hline Compuesto & $\delta(-\mathrm{CH}=\mathrm{N})$ & $\delta(-\mathrm{NH})$ & $\delta\left(\mathrm{NR}_{\mathbf{2}}\right)$ & $\delta$ (furano, aromático) \\
\hline $\operatorname{Pd}\left(\mathrm{LF}^{3}\right)_{2}$ & $8,55(\mathrm{~s}, 2 \mathrm{H})$ & - & $8,21,8,72\left(\mathrm{~d}, \mathrm{a}, 4 \mathrm{H}, \mathrm{NH}_{2}\right)$ & $\begin{array}{c}7,49\left(\mathrm{~d}, 2 \mathrm{H}^{3}\right) \\
6,89\left(\mathrm{~d}, 2 \mathrm{H}^{4}\right) \\
3,30\left(\mathrm{c},-\mathrm{CH}_{2}\right) \\
1,85\left(\mathrm{t},-\mathrm{CH}_{3}\right)\end{array}$ \\
\hline $\mathrm{Pd}\left(\mathrm{LF}^{4}\right)_{2}$ & $8,01(\mathrm{~s}, 2 \mathrm{H})$ & - & 9,86 (s, 2H, NHPh) & $\begin{array}{c}6,70\left(\mathrm{dd}, 2 \mathrm{H}^{3}\right) \\
6,74\left(\mathrm{dd}, 2 \mathrm{H}^{4}\right) \\
7,95\left(\mathrm{~d}, 2 \mathrm{H}^{5}\right) \\
7,09\left(\mathrm{t}, 2 \mathrm{H}_{\text {meta }}, \mathrm{Ph}\right) \\
7,35\left(\mathrm{t}, 4 \mathrm{H}_{\text {para }}, \mathrm{Ph}\right) \\
7,52\left(\mathrm{dd}, 4 \mathrm{H}_{\text {orto }}, \mathrm{Ph}\right)\end{array}$ \\
\hline$P d\left(L F^{5}\right) 2$ & $7,89(\mathrm{~s}, 2 \mathrm{H})$ & - & $\begin{array}{r}9,54(\mathrm{~s}, 2 \mathrm{H}, \mathrm{NHPh}) \\
7,7 \\
7 \\
7\end{array}$ & $\begin{array}{c}6,87\left(\mathrm{t}, \mathrm{H}^{4}\right) \\
6,98\left(\mathrm{~d}, \mathrm{H}^{3}\right) \\
, 13,7,06(\mathrm{~m}, 10 \mathrm{H}, \mathrm{C}-\mathrm{Ph}) \\
7,19\left(\mathrm{t}, 4 \mathrm{H}_{\text {meta }}, \mathrm{N}-\mathrm{Ph}\right) \\
7,38\left(\mathrm{~d}, 2 \mathrm{H}_{\text {para }}, \mathrm{N}-\mathrm{Ph}\right) \\
7,85\left(\mathrm{~d}, 4 \mathrm{H}_{\text {orto }}, \mathrm{N}-\mathrm{Ph}\right)\end{array}$ \\
\hline $\mathrm{Pd}\left(\mathrm{LF}^{6}\right)_{2}$ & $8,05(\mathrm{~s}, 1 \mathrm{H})$ & - & $9,89(\mathrm{~s}, 2 \mathrm{H}, \mathrm{NHPh})$ & $\begin{array}{c}7,12\left(\mathrm{~d}, \mathrm{H}^{4}\right) \\
7,23\left(\mathrm{~d}, \mathrm{H}^{3}\right) \\
2,08\left(\mathrm{c},-\mathrm{CH}_{2}\right) \\
7,36\left(\mathrm{t}, 2 \mathrm{H}_{\text {meta }}, \mathrm{N}-\mathrm{Ph}\right) \\
7,52\left(\mathrm{t}, 1 \mathrm{H}_{\text {para }}, \mathrm{N}-\mathrm{Ph}\right) \\
7,84\left(\mathrm{~d}, 2 \mathrm{H}_{\text {orto }}, \mathrm{N}-\mathrm{Ph}\right)\end{array}$ \\
\hline
\end{tabular}

$\mathrm{s}=$ singlete, $\mathrm{d}=$ doblete, $\mathrm{t}=$ triplete, $\mathrm{c}=$ cuarteto, $\mathrm{a}=$ ancha

\subsection{Actividad biológica}

La citotoxicidad de los extractos fue evaluada en diferentes líneas celulares tumorales empleando el método de la sulforodamina B (SRB). Las cuatro líneas de células tumorales de humano ensayadas fueron las siguientes: adenocarcinoma de colon (HT-29), carcinoma de pulmón (H460), adenocarcinoma de mama (MCF7) y leucemia mielogénica crónica (K562). Las células se sembraron en el medio de cultivo DMEM (medio Dulbecco modificado por Eagle) a $37^{\circ} \mathrm{C}$ y complementado con suero fetal de bovino al 10\% [FBS] (Difco), $100 \mathrm{U} \mathrm{mL}^{-1} \mathrm{de}$ penicilina, $100 \mathrm{mg} \mathrm{mL}^{-1}$ de gentamicina y L-glutamina $2 \mathrm{mM}^{8}$

8 Sheham, P. et al. J. Natl Canler Inst. 82, 1990, p. 1107. 


\section{DISCUSIÓN DE RESULTADOS}

\subsection{Espectroscopía de infrarrojo (IR)}

En todos los espectros IR, las bandas de absorción observadas en el rango de $3130-3195 \mathrm{~cm}^{-1}$ fueron asignadas al grupo N-H para los ligantes furaldehído tiosemicarbazona (LF). Estas bandas desaparecen después de la formación de los complejos de paladio(II) y cobre(II). Esta evidencia confirma la desprotonación del grupo tioamido (-NHCS) en los ligantes y la deslocalización electrónica entre el grupo tiocarbonilo $(\mathrm{C}=\mathrm{S})$ y el grupo imino $(\mathrm{C}=\mathrm{N})$. Las bandas de absorción de los grupos imino, $\mathrm{n}(\mathrm{C}=\mathrm{N})$ y tiocarbonilo, $\mathrm{n}(\mathrm{C}=\mathrm{S})$, observadas en los ligantes orgánicos, se desplazan hacia frecuencias menores en el rango de $5-20 \mathrm{~cm}^{-1}$ y $20-90 \mathrm{~cm}^{-1}$, respectivamente, en la formación de los complejos metálicos. Este resultado confirma la coordinación de los átomo dadores nitrógeno y azufre hacia el ion paladio(II) y cobre(II). Además, los espectros IR revelan que las bandas de absorción correspondiente a los grupos -aminos terminales $\left(\mathrm{NH}_{2} \mathrm{y} \mathrm{NHPh}\right)$ permanecen invariables tanto en los ligantes como en la formación de los complejos de paladio(II) y cobre(II). Esta evidencia indica que los grupos aminos terminales no se encuentran coordinados con el centro metálico. ${ }^{9}$

\subsection{Espectros de $\mathrm{RMN}^{-1} \mathrm{H}$ y $\mathrm{RMN}^{-13} \mathrm{C}$}

Las asignaciones de las resonancias de ${ }^{1} \mathrm{H}$ y ${ }^{13} \mathrm{C}$ para los ligantes derivados del furaldehído tiosemicarbazona y sus complejos de paladio(II), fueron obtenidas a partir de los espectros de $\mathrm{RMN}\left({ }^{1} \mathrm{H}\right.$ y $\left.{ }^{13} \mathrm{C}\right)$ registrados en DMSO-d 6 a $300 \mathrm{~K}$.

En los espectros de $\mathrm{RMN}-{ }^{1} \mathrm{H}$ de los ligantes, las señales de los protones $=\mathrm{N}-\mathrm{NH}$ aparecen como singletes en el rango de 10.6-11.97 ppm, tal como se observa para el ligante $\mathrm{LF}^{4}(\delta=11.97 \mathrm{ppm})$ (Fig. 7). Estas señales desaparecen en los espectros $\mathrm{RMN}-{ }^{1} \mathrm{H}$ de los complejos de paladio(II) (Fig. 8), indicando la desprotonación del grupo $=\mathrm{N}-\mathrm{NH}$. Las señal del protón imino $\mathrm{HC}=\mathrm{N}$ que aparece en los ligantes como singlete en el rango de 7.78 - $8.10 \mathrm{ppm}$, se desplaza entre $0.04-0.77$

9 Hernández, W. Bioinorg. Chem. Apll. 1, 2003, p. 271. 
ppm hacia campo bajo en los espectros de los complejos de paladio(II). Esta resultado revela la coordinación del nitrógeno imino al ión paladio(II) (Seleem, 2005; 1003). ${ }^{10}$

Figura 7

Espectro de $\mathrm{RMN}-{ }^{-1} \mathrm{H}$ del ligante $\mathrm{LF}^{4}$

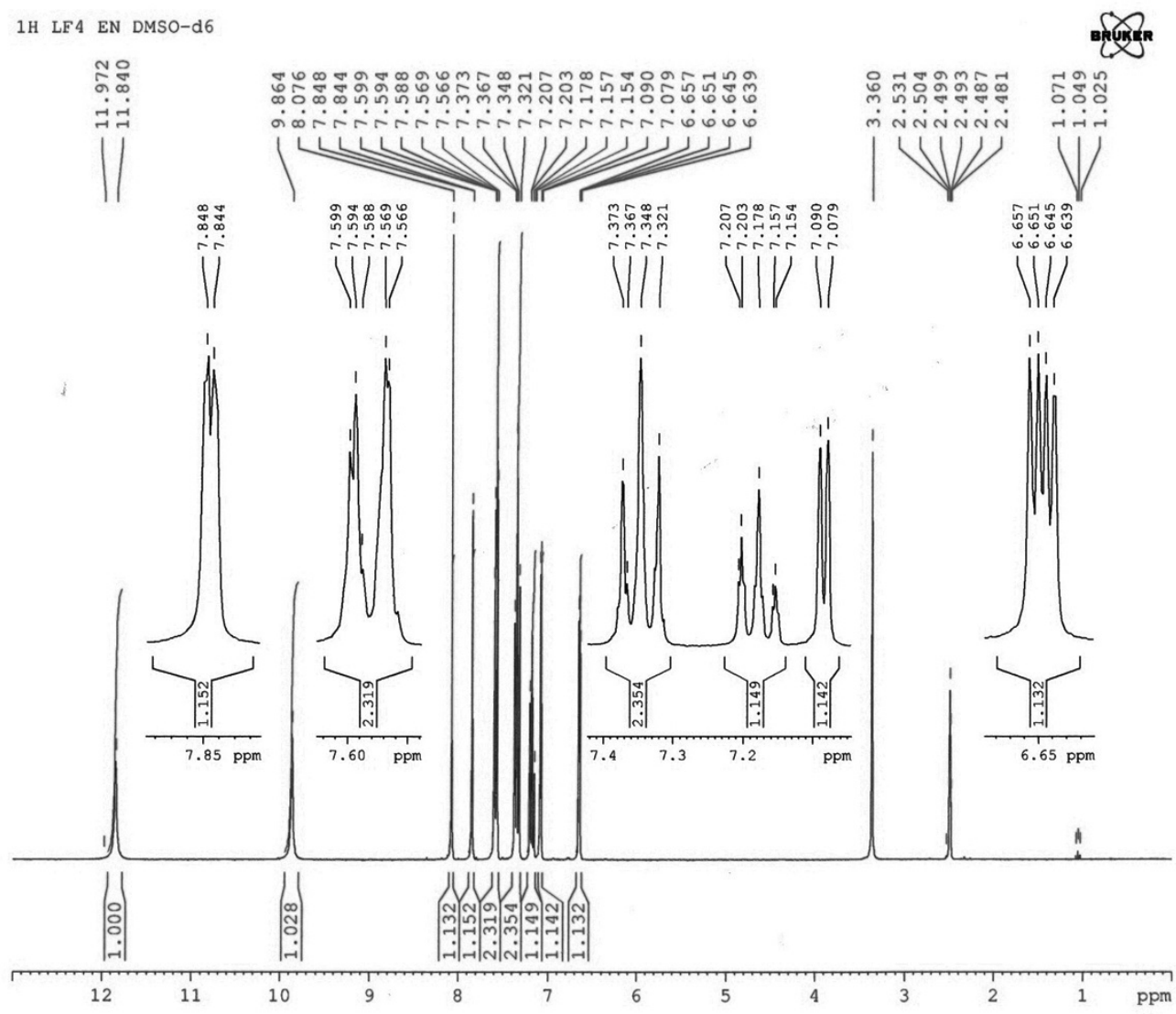

10 Seleem, H. et al. J. coord. chem 58, 2005, p. 2003. 
Hernández, Paz, Vaisberg, Spodine, Richter, Beyer

Figura 8

Espectro de $\mathrm{RMN}-{ }^{-1} \mathrm{H}$ del complejo $\mathrm{Pd}\left(\mathrm{LF}^{4}\right)_{2}$

1H PdLF4 EN DMSO-d6 $25.6 \mathrm{mg}$
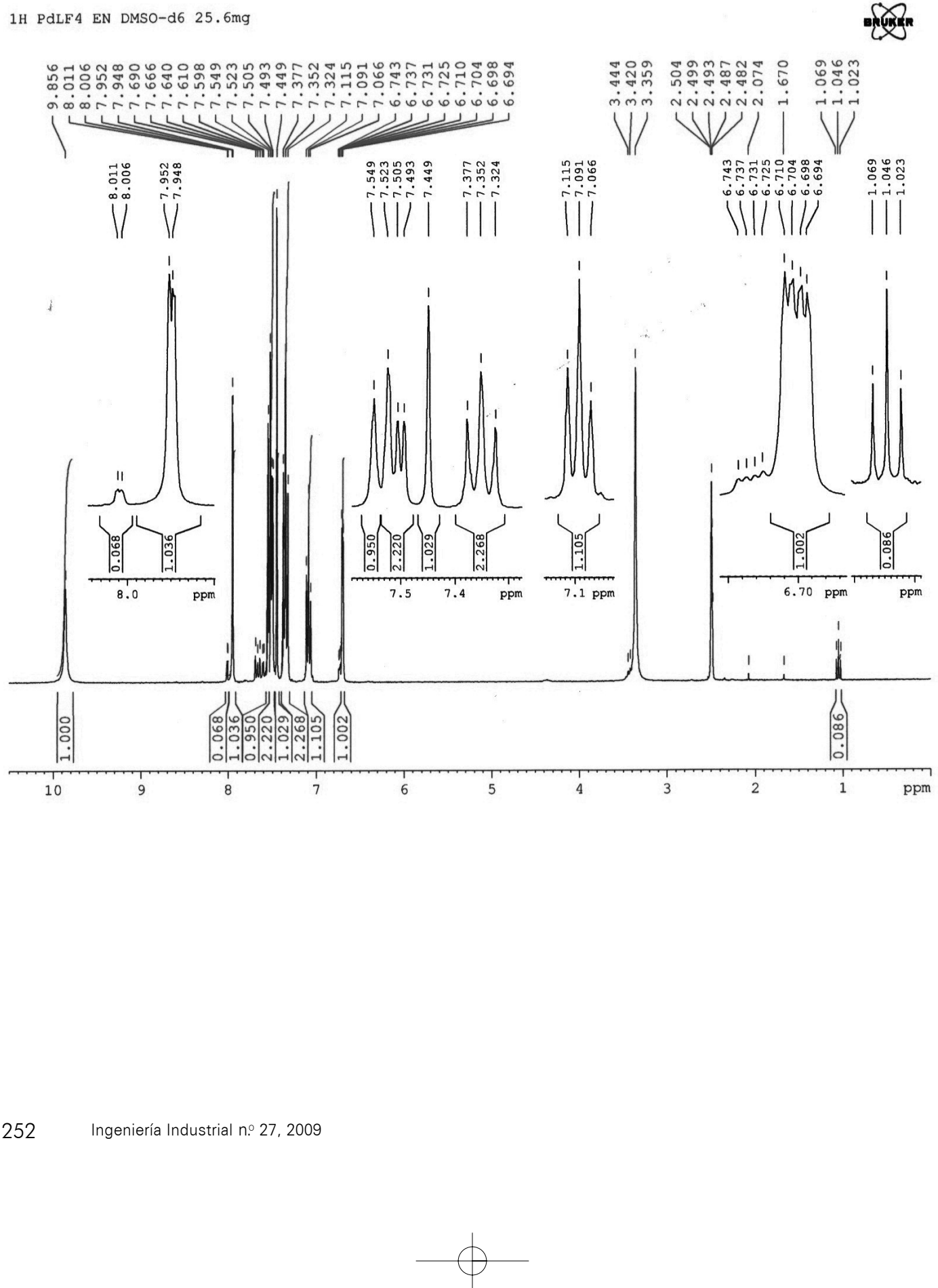
Las señales de los protones aromáticos de la amina terminal y del anillo furano aparecen de acuerdo con su multiplicidad calculada y dentro del rango establecido de $7.18-7.83$ y $6.24-7.85$ ppm, respectivamente. Estas señales no sufren cambios relevantes en los desplazamientos químicos para los complejos de paladio(II). La presencia del grupo fenilo en la amina terminal (NHPh) de los ligantes $\mathrm{LF}^{4}-\mathrm{LF}^{6}$, produce un desplazamiento químico de aproximadamente $2.0 \mathrm{ppm}$ hacia campo bajo con respecto al grupo $-\mathrm{NH}_{2}$ presente en los ligantes $\mathrm{LF}^{1}-\mathrm{LF}^{3}$. Este resultado indica que los ligantes $\mathrm{LF}^{1}-\mathrm{LF}^{3}$ son ligeramente menos básicos que los ligantes $\mathrm{LF}^{4}-\mathrm{LF}^{6}$. Debido a la no existencia de cambios relevantes en las señales de los grupos $-\mathrm{NH}_{2}$ ó $\mathrm{NHPh}$ en los complejos de paladio(II), se confirma que estos grupos no se coordinan al ión paladio(II). ${ }^{11}$

En los espectros de $\mathrm{RMN}-{ }^{13} \mathrm{C}$ de los ligantes orgánicos, la señal de resonancia que se observa en el rango de 132.47-133.45 ppm se asigna al grupo $\mathrm{HC}=\mathrm{N}$; estos resultados son similares a los desplazamientos químicos encontrados para los ligantes tiofeno tiosemicarbazona y pirrol tiosemicarbazona. Las señales de los grupos tiocarbonilos $\mathrm{C}=\mathrm{S}$ se observan en el rango de 174.49-175.65 ppm, mientras que las líneas de resonancia de los carbonos aromáticos aparecen en el rango de $125.49-139.04$ ppm. $^{12}$

\subsection{Actividad biológica}

La actividad antitumoral in vitro de los ligantes tiosemicarbazona $\left(\mathrm{LF}^{1-7}\right)$ y los complejos de paladio(II), $\mathrm{Pd}\left(\mathrm{LF}^{4-7}\right)_{2}$; y cobre(II), $\mathrm{Cu}\left(\mathrm{LF}^{3-6}\right)_{2}$ fue evaluada en diferentes líneas de células tumorales de humano, después de 72 horas de haber sido sembradas en el medio de cultivo con cada uno de los ligantes o sus respectivos complejos de paladio(II) y cobre(II).

Las actividades citotóxicas in vitro de los ligantes orgánicos revelaron menor citotoxicidad $\left(\mathrm{CI}_{50}>20 \mu \mathrm{M}\right)$ al ser evaluadas frente a las diferentes líneas de células tumorales de humano; tal como se obser-

11 Yakuphanoglu, F. et al. Synth React. Inorg. Met. Org. Chem. 32, 2002, p. 1865.

12 Chandra, S. et al. Synth React. Inorg. Met. Org. Chem. 31, 2001, p. 147. 
va en la tabla 7, la actividad citotóxica se incrementa cuando los ligantes derivados del furaldehído tiosemicarbazona se encuentran coordinados con los metales de paladio y cobre. Los complejos de paladio(II), $\operatorname{Pd}\left(\mathrm{LF}^{4}\right)_{2}$ y $\mathrm{Pd}\left(\mathrm{LF}^{5}\right)_{2}$ mostraron mayor citotoxicidad $\left(\mathrm{CI}_{50}=0.28-1.23\right.$ $\mu \mathrm{M})$ que los demás complejos metálicos $\left(\mathrm{CI}_{50}=5.14-28.29 \mu \mathrm{M}\right)$ frente a las líneas de células tumorales de humano: H460, MCF7, K562 y HT29. Además, el complejo de paladio(II), $\mathrm{Pd}\left(\mathrm{LF}^{5}\right)_{2}\left(\mathrm{CI}_{50}=0.28-0.29\right.$ $\mu \mathrm{M})$ resultó ser más citotóxico que el complejo de paladio(II) con ligante 5-cloro tiofeno tiosemicarbazona, $\mathrm{Pd}\left(\mathrm{TSC}^{4}\right)_{2} \quad\left(\mathrm{CI}_{50}=9.10-13.65 \mu \mathrm{M}\right)$ evaluado in vitro frente a las líneas de células tumorales de humano MCF-7 y HT29. Probablemente, la lipofilicidad y la geometría plana del complejo $\mathrm{Pd}\left(\mathrm{LF}^{5}\right)_{2}$, con un grupo sustituyente fenilo en el anillo furano y en la amina terminal incrementan la citotoxicidad del complejo al intercalarse entre las bases purinas y pirimidinas del $\mathrm{ADN}$ celular tumoral. Por otro lado, el complejo de paladio(II), $\mathrm{Pd}\left(\mathrm{LF}^{5}\right)_{2}$ $\left(\mathrm{CI}_{50}=0.28 \mu \mathrm{M}\right)$ presentó mayor actividad antiproliferante que los ligantes 1,2-naftoquinona ( $\mathrm{NQ})\left(\mathrm{CI}_{50}=14.58 \mu \mathrm{M}\right)$ y 1,2-naftoquinona tiosemicarbazona(NQTS) $\left(\mathrm{CI}_{50}=3.50 \mu \mathrm{M}\right)$ y su complejo de paladio(II), $\mathrm{Pd}(\mathrm{NQTS}) \mathrm{Cl}\left(\mathrm{CI}_{50}=9.75 \mu \mathrm{M}\right)$ (figura 9$)$ evaluados in vitro frente a la línea celular tumoral MCF-7. ${ }^{13}$

\section{Figura 9}

Ligantes orgánicos derivados de la naftoquinona y su complejo de paladio(II)<smiles>O=C1C=Cc2ccccc2C1=O</smiles>

(NQ)<smiles>NC(=S)N/N=C1\C=Cc2ccccc2C1=O</smiles>

(NQTS)<smiles>NC1=NN2c3ccc4ccccc4c3O[Pb]2(Cl)S1</smiles>

Complejo Pd(NQTS)Cl

Entre los complejos de cobre(II) ensayados, el complejo $\mathrm{Cu}\left(\mathrm{LF}^{3}\right)_{2}$ presentó mayor citotoxicidad $\left(\mathrm{CI}_{50}=6.87-7.45 \mu \mathrm{M}\right)$ que los demás complejos de cobre(II) $\left(\mathrm{CI}_{50}=22.18-28.29 \mu \mathrm{M}\right)$ frente a las diferentes

13 Afrasiabi, Z. et al. Inorg. Chim. Acta, 2004, p. 271 
líneas de células tumorales de humano. Además, el complejo de cobre(II), $\mathrm{Cu}\left(\mathrm{LF}^{3}\right)_{2}$ mostró mayor actividad citotóxica que el complejo $\operatorname{Pd}\left(\mathrm{TSC}^{4}\right)_{2}$ con ligante tiofeno tiosemicarbazona, evaluado in vitro frente a las líneas de células tumorales de humano MCF-7 y HT29. Este resultado indica que la naturaleza del ligante orgánico y del metal son factores importantes en la inhibición del crecimiento celular tumoral.

Tabla 7

Valores de $\mathrm{Cl}_{50}(\mu \mathrm{M})^{\mathrm{a}}$ para los complejos de paladio(II) y cobre(II) con ligandos derivados del furaldehído tiosemicarbazona en diferentes líneas de células tumorales de humano

\begin{tabular}{lrrrr}
\hline $\begin{array}{l}\text { Línea celular/ H460 } \\
\text { compuesto }\end{array}$ & MCF7 & k562 & HT29 \\
\hline $\mathrm{Pd}\left(\mathrm{LF}^{4}\right)_{2}$ & 1,23 & 1,15 & 0,30 & 1,05 \\
$\mathrm{Pd}\left(\mathrm{LF}^{5}\right)_{2}$ & 0,32 & 0,28 & 0,31 & 0,29 \\
$\mathrm{Pd}\left(\mathrm{LF}^{6}\right)_{2}$ & 24,00 & 21,45 & 22,85 & 23,06 \\
$\mathrm{Pd}\left(\mathrm{LF}^{7}\right)_{2}$ & 22,63 & 20,68 & 5,65 & 5,14 \\
$\mathrm{Cu}\left(\mathrm{LF}^{3}\right)_{2}$ & 7,08 & 6,93 & 7,45 & 6,87 \\
$\mathrm{Cu}\left(\mathrm{LF}^{4}\right)_{2}$ & 28,29 & 25,65 & 27,94 & 26,35 \\
$\mathrm{Cu}\left(\mathrm{LF}^{5}\right)_{2}$ & 22,18 & 23,53 & 24,07 & 22,85 \\
$\mathrm{Cu}\left(\mathrm{LF}^{6}\right)_{2}$ & 23,82 & 22,56 & 23,45 & 24,35 \\
\hline
\end{tabular}

${ }^{\mathrm{a}} \mathrm{CI}_{50}$ corresponde a la concentración requerida para inhibir al 50\% el crecimiento celular cuando las células son expuestas a los compuestos durante 48 horas. Cada valor es el promedio de dos experimentos independientes.

\section{CONCLUSIONES}

Del estudio realizado se puede concluir lo siguiente:

- Los datos analíticos y espectroscópicos confirman las fórmulas estructurales propuestas de los ligantes furaldehído tiosemicarbazona y de los complejos de paladio(II) y cobre(II) preparados.

- Los ligantes orgánicos se encuentran coordinados a los metales paladio y cobre a través de sus átomos dadores de nitrógeno (grupo imino) y azufre (grupo tiocarbonilo).

- Los complejos metálicos de paladio(II) y cobre(II) presentaron mayor citotoxicidad que sus ligantes orgánicos. Dentro de los complejos de paladio(II), los complejos $\operatorname{Pd}\left(\mathrm{LF}^{4}\right)_{2} \mathrm{y} \operatorname{Pd}\left(\mathrm{LF}^{5}\right)_{2}$, fueron más ci- 
totóxicos frente a las diferentes líneas de células tumorales de humano ensayadas mientras que el complejo $\mathrm{Cu}\left(\mathrm{LF}^{3}\right)_{2}$ presentó mayor citotoxicidad in vitro con respecto a los demás complejos de cobre(II).

\section{BIBLIOGRAFÍA}

Afrasiabi, Z. et al. Inorg. Chim. Acta 357, 2004.

Brozowski, Z. et al. European Journal of Medicinal Chemistry 42, 2007.

Chandra, S. et al. Synth. React. Inorg. Met. Org. Chem., 31, 2001.

Hall, I. et al. Pharmazie 55, 2000.

Hernández, W. Bioinorg. Chem. Appl., 2008.

—. Bioinorg. Chem. Appl. 1, 2003.

Lobana, T. et al. J. Chem. Doc., Dalton Trans., 1997.

Morris, J. et al. Cancer Research 29, 1969.

Newman, R. et al. Journal of Pharmaceutical Sciences 71, 1981.

Seleem, H. et al. J. Coord. Chem. 58, 2005.

Skehan, P. et al. J. Natl Cancer Inst. 82, 1990.

Yakuphanoglu, F. et al. Synth. React. Inorg. Met. Org. Chem. 32, 2002.

\section{Glosario de términos}

Ligantes: Compuestos orgánicos que se coordinan a los metales de transición a través de los átomos de oxígeno, azufre o nitrógeno.

Complejo metálico: Compuesto que presenta uno o más ligantes orgánicos coordinados al centro metálico a través de sus átomos dadores.

Bandas de absorción: Señales características del grupo funcional orgánico que se observan en un espectro de infrarrojo.

Líneas de resonancia: Señales correspondientes a los hidrógenos o carbonos presentes en los compuestos preparados y que se observan a partir de un espectro de resonancia magnética nuclear. 
Línea celular tumoral: Diferentes tipos de células tumorales: adenocarcinoma de colon, carcinoma de pulmón, adenocarcinoma de mama y leucemia mielogénica crónica.

Actividad citotóxica: Es la potencialidad que presentan los compuestos para disminuir el crecimiento de las células tumorales luego de haber sido cultivados en las diferentes líneas celulares tumorales.

In vitro: Acción que presentan los compuestos sobre células vivas cultivadas en placas de cultivo.

Valor $\mathrm{CI}_{50}$ : Concentración micromolar necesaria para disminuir el crecimiento celular tumoral al $50 \%$. 